\title{
Cooperative Beamforming, Power Allocation and Relay Selection in MIMO Cognitive Radio Systems
}

\author{
M. Abbasi-Jannatabad ${ }^{1,2,3}$ and H. Khoshbin ${ }^{1}$ \\ ${ }^{1}$ Electrical Engineering Department, Ferdowsi University of Mashhad \\ ${ }^{2}$ Khorasan Institute of Higher Education, Mashhad, Iran \\ ${ }^{3}$ Communications and Computer Research Center, Ferdowsi University of Mashhad
}

\begin{abstract}
Cognitive radio technology can effectively improve spectral efficiency by allowing secondary users to access licensed bands without any harmful interference to primary users. Due to harmful channel effects such as fading, or because of far distance between secondary transmitter and receiver, some secondary users who don't have any message to transmit at the time, can act as relay nodes to improve secondary users' connectivity. In this paper, a new algorithm is proposed for cooperative beamforming, power allocation and relay selection in the multiple input multiple output (MIMO) cognitive radio systems where a pair of secondary user communicates with each other assisted by a set of single antenna relay nodes. The objective is to maximize signal to interference plus noise ratio of secondary user subject to guarantee the primary user's quality of service and power constraints of secondary user and relay node. The transmitter and receiver beamforming vectors of primary user and secondary user are estimated by the proposed two-step iterative algorithm. The performance of the algorithm is evaluated through simulations.
\end{abstract}

Index Terms - relay cognitive radio, MIMO systems, cooperative beamforming, power allocation, relay selection.

\section{INTRODUCTION}

Spectrum usage efficiency can be improved using cognitive radio (CR) technology in which secondary users (SUs) are allowed to access licensed bands that are originally allocated to primary network without causing any harmful interference to primary users (PUs) [1]. There are two main secondary access schemes to licensed bands. The first approach is based on opportunistic communication in which SUs can access the licensed bands only when PUs are absent. This approach is called "interweave" technique. In the second scheme, SUs are allowed to access the licensed bands simultaneously with PUs [2]. In the later spectrum usage efficiency improved compared to the former.

To increase the percentage of spectrum usage, some SUs who don't have any message to transmit at the time, can utilized as relay nodes. These relay nodes cooperate with SU transmitters to relay messages to desired destinations. In recent years, many relay schemes are investigated. In [3] resource allocation in relay OFDM-based CR system is considered based on decode-and-forward (DF) protocol. The dual decomposition technique is adopted to obtain an asymptotically optimal subcarrier pairing, relay selection and power allocation. The optimization problem is based on maximization of sum rate subject to interference limit for PU and power constraints in SU transmitter and relays. In [4] authors investigate power and channel allocation for cooperative relay in a three-node CR network. Three nodes are considered which consists of a source, a relay and a destination. Three end-to-end possible communications are assumed based on three channels consists of direct channel, dual-hop channel and relay channel. The resources are allocated based on maximization of channel capacity under PU's interference and power constraints in source and relay nodes. In [5] the problem of relay and power allocation for OFDM-based CR systems with single antenna are considered where the capacity of SU employing relays is maximized subject to total transmission power constraint and interference limit for PU. Due to high computational complexity of the optimization problem, three sub-optimal schemes are presented. The authors in [6] investigate the problem of resource (subcarrier and power) allocation in an OFDMA-based relayed cellular cognitive radio network in which a base station (BS) services some user equipment (UE) via multiple relay stations (RSs). The resource allocation problem must decide for each symbol which subcarrier at which RSs and at what power level would relay. The objective function is maximization of network capacity. Joint relay selection and power allocation are investigated in [7] to maximize system throughput with limited interference to PUs in CR network. The authors develop an optimal approach based on dual method and then propose a suboptimal algorithm to reduce computational complexity. In [8] the authors present an optimal scheme for power allocation and relay selection in CR networks where a pair of cognitive (secondary) transceiver communicates with each other using some two-way relay nodes. The goal of the proposed power allocation and relay selection algorithm is to maximize the achievable rate subject to interference constraint of PU and power constraints of cognitive transceivers and relay node. In [9] a simplified power allocation algorithm is proposed for cognitive multi-node relay networks. The optimization problem is the maximization of secondary system capacity under the interference on the PU and the maximum transmission power constraint. A beamforming and power control scheme is proposed for an overlay CR network in [10]. The proposed algorithm minimizes the total power consumed by the network while satisfying each user's SINR requirement. The authors proposed an iterative algorithm based on second-order cone programming and demonstrated that the algorithm greatly improves the overall power saving of the network. 


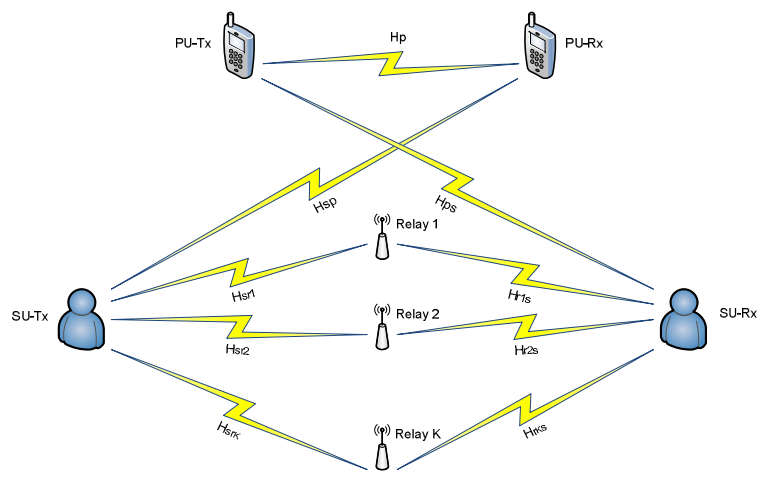

Fig. 1 System model

In this paper, we propose a new algorithm for cooperative beamforming, power allocation and relay selection in MIMOCR systems. In this system relay nodes cooperate to deliver SU transmitter's message to SU receiver. Relay nodes act based on amplify-and forward (AF) protocol. The proposed algorithm is developed under a criterion with two constraints. In the criterion of the algorithm, sum signal to interference plus noise (SINR) of SU is maximized subject to 1) maximum allowable transmit power of SU and relay node and 2) a minimum threshold for PU's SINR in order to guarantee the required PU's QoS. A two-step iterative algorithm estimates the transmitter and receiver beamforming vectors of PU and SU by maximizing the sum SINR of SU and also computes allocated powers to the PU, SU and relay node by applying the given two constraints. In the following, a MIMO relay CR system is modeled based on a pair of PU transmitter (PU-Tx) and PU receiver (PU-Rx), a pair of SU transmitter (SU-Tx) and SU receiver (SU-Rx) and also $K$ relay nodes in Section II. The proposed algorithm is developed in Section III. Computer simulation results are presented in Section IV and Section V concludes the paper.

\section{SYSTEM MODEL}

A MIMO relay CR system with a pair of PU transmitter (PU-Tx) and PU receiver (PU-Rx), a pair of SU transmitter (SU-Tx) and SU receiver (SU-Rx) and also $K$ relay nodes is shown in Fig. 1 where the PU-Tx, PU-Rx, SU-Tx and SU-Rx are equipped by antenna arrays with $N_{p}, M_{p}, N_{s}$ and $M_{s}$ elements, respectively. Each relay node is equipped with single antenna. The communication between SU-Tx and SU-Rx takes place in two time slots. In the first slot, SU-Tx transmits its data to relay nodes. When $s_{p}$ and $s_{s}$ are transmitted symbols for PU and SU, respectively, the received signals of $k$-th relay node $y_{r_{k}}$, is given as

$y_{r_{k}}=\mathbf{H}_{s r_{k}} \mathbf{v}_{s} s_{s}+\mathbf{H}_{p r_{k}} \mathbf{v}_{p} s_{p}+n_{r_{k}}$

where $s_{p}$ and $s_{s}$ are normalized energy signals with independent and identically distributions (i.i.d.). Also $\mathbf{H}_{s r_{k}}$ represents the channel between the SU-Tx and relay and $\mathbf{H}_{p r_{k}}$ is the channel between the PU-Tx and relay. Moreover $\mathbf{v}_{p}$ and $\mathbf{v}_{s}$ are PU's and SU's transmit beamforming vectors, respectively, such that $p_{p}=\mathbf{v}_{p}^{H} \mathbf{v}_{p}$ and $p_{s}=\mathbf{v}_{s}^{H} \mathbf{v}_{s}$ are transmitted power of PU and SU, respectively. Moreover $n_{r_{k}}$ is zero mean additive white Gaussian noise (AWGN). Meanwhile, $E\left[n_{r_{k}} n_{r_{k}}^{H}\right]=N_{r_{k}}$.

In the second time slot, the selected relay node retransmits the amplified version of its received signal in the first time slot, $s_{r_{k}}=\sqrt{P_{r_{k}}} y_{r_{k}}$ to SU-Rx. The received signals of PU, $\mathbf{y}_{p}$ and $\mathrm{SU}, \mathbf{y}_{s}$, ( after applying $\mathbf{u}_{p}$, PU's receiver beamforming vector, $\mathbf{u}_{s}$ and SU's receiver beamforming vector and $\mathbf{u}_{r_{k}}$ ) are given as

$$
\begin{aligned}
& \mathbf{y}_{p}=\mathbf{u}_{p}^{H} \mathbf{H}_{p} \mathbf{v}_{p} s_{p}+\mathbf{u}_{p}^{H} \mathbf{H}_{s p} \mathbf{v}_{s} s_{s}+\mathbf{u}_{p}^{H} \mathbf{H}_{r_{k} p} s_{r_{k}}+\mathbf{u}_{p}^{H} \mathbf{n}_{p} \\
& \mathbf{y}_{s}=\mathbf{u}_{s}^{H} \mathbf{H}_{r_{k} s} s_{r_{k}}+\mathbf{u}_{s}^{H} \mathbf{H}_{p s} \mathbf{v}_{p} s_{p}+\mathbf{u}_{s}^{H} \mathbf{n}_{s}
\end{aligned}
$$

where $\mathbf{H}_{p}$ is the channel between the PU-Tx and the PU-Rx , $\mathbf{H}_{s p}$ is the channel between the SU-Tx and the PU-Rx, $\mathbf{H}_{r_{k} p}$ is the channel between the relay and the PU-Rx. $\mathbf{H}_{r_{k} s}$ denotes the channel between the relay and the SU-Rx and $\mathbf{H}_{p s}$ is the channel between the PU-Tx and the SU-Rx. Also $\mathbf{n}_{p}$ and $\mathbf{n}_{s}$ are zero mean AWGN vectors, respectively. Meanwhile, $E\left[\mathbf{n}_{p} \mathbf{n}_{p}^{H}\right]=N_{p} \mathbf{I}_{M_{p}}$ and $E\left[\mathbf{n}_{s} \mathbf{n}_{s}^{H}\right]=N_{s} \mathbf{I}_{M_{s}}$ where $\mathrm{I}_{N}$ is $N \times N$ unitary matrix and $(.)^{H}$ represents complex conjugate operation.

$$
\begin{aligned}
\operatorname{SINR}_{\mathrm{PU}}= & \frac{\mathbf{v}_{p}^{H}\left(\mathbf{H}_{p}^{H}+\sqrt{P_{r_{k}}} \mathbf{H}_{p r_{k}}^{H} \mathbf{H}_{r_{k} p}^{H}\right) \mathbf{u}_{p} \mathbf{u}_{p}^{H}\left(\mathbf{H}_{p}+\sqrt{P_{r_{k}}} \mathbf{H}_{r_{k} p} \mathbf{H}_{p r_{k}}\right) \mathbf{v}_{p}}{\mathbf{v}_{s}^{H}\left[\left(\mathbf{H}_{s p}^{H}+\sqrt{P_{r_{k}}} \mathbf{H}_{s r_{k}}^{H} \mathbf{H}_{r_{k} p}^{H}\right) \mathbf{u}_{p} \mathbf{u}_{p}^{H}\left(\mathbf{H}_{s p}+\sqrt{P_{r_{k}}} \mathbf{H}_{r_{k} p} \mathbf{H}_{s r_{k}}\right)+\left(\frac{N_{p}+P_{r_{k}} N_{r_{k}}}{P_{s}}\right) \mathbf{I}_{N_{s}}\right] \mathbf{v}_{s}} \\
\operatorname{SINR}_{\mathrm{SU}}= & \frac{P_{r_{k}} \mathbf{v}_{s}^{H} \mathbf{H}_{s r_{k}}^{H} \mathbf{H}_{r_{k} s}^{H} \mathbf{u}_{s} \mathbf{u}_{s}^{H} \mathbf{H}_{r_{k} s} \mathbf{H}_{s r_{k}} \mathbf{v}_{s}}{\mathbf{v}_{p}^{H}\left[\left(P_{r_{k}} \mathbf{H}_{p r_{k}}^{H} \mathbf{H}_{r_{k} s}^{H} \mathbf{u}_{s} \mathbf{u}_{s}^{H} \mathbf{H}_{r_{k} s} \mathbf{H}_{p r_{k}}+\mathbf{H}_{p s}^{H} \mathbf{u}_{s} \mathbf{u}_{s}^{H} \mathbf{H}_{p s}\right)+\left(\frac{\left.N_{s}+P_{r_{k}} N_{r_{k}}\right)}{P_{p}}\right) \mathbf{I}_{N_{p}}\right] \mathbf{v}_{p}}
\end{aligned}
$$


Based on (1)-(3), by substituting (1) into (2) and (3), the signal to interference plus noise ratio (SINR) of the PU, $\mathrm{SINR}_{\mathrm{PU}}$ and the SINR of SU, SINR ${ }_{\mathrm{SU}}$, are defined as (4) and (5), in the bottom of the previous page, respectively, where $N_{p}=E\left[\mathbf{u}_{p}^{H} \mathbf{n}_{p} \mathbf{n}_{p}^{H} \mathbf{u}_{p}\right] \quad, \quad N_{s}=E\left[\mathbf{u}_{s}^{H} \mathbf{n}_{s} \mathbf{n}_{s}^{H} \mathbf{u}_{s}\right] \quad$ and $N_{r_{k}}=E\left[\mathbf{u}_{r_{k}}^{H} \mathbf{n}_{r_{k}} \mathbf{n}_{r_{k}}^{H} \mathbf{u}_{r_{k}}\right]$.

\section{COOPERATIVE BEAMFORMING AND POWER ALLOCATION ALGORITHM}

In this section, a new cooperative beamforming, power allocation and relay selection algorithm is proposed to improve spectrum usage efficiency in MIMO-relay CR system. The algorithm is developed based on a criterion in which SINR of SU is maximized under power constraints of SU transmitter and relay node while the PU's QoS is guaranteed by means of defining a threshold for $\mathrm{SINR}_{\mathrm{PU}}$. The criterion of the proposed algorithm is defined based on maximizing the SINR of SU subject to a maximum transmitted power of $\mathrm{SU}, P_{s}^{\max }$, a maximum transmitted power of relay node, $P_{r}^{\max }$ and the threshold of $\mathrm{SINR}_{\mathrm{PU}}, \gamma_{p}$. The optimization problem is formulated as follows.

$C=\underset{\mathbf{v}_{s}, \mathbf{u}_{s}, \mathbf{v}_{p}, \mathbf{u}_{p}}{\arg \max }\left\{\mathrm{SINR}_{\mathrm{SU}}\right\}, \quad$ s.t. $\left\{\begin{array}{l}\mathrm{SINR}_{\mathrm{PU}} \geq \gamma_{p} \\ p_{s} \leq P_{s}^{\max } \\ p_{r_{k}} \leq P_{r}^{\max } \quad \text { for } \mathrm{k}=1, \cdots, K\end{array}\right.$

We employ a two-step algorithm in order to maximize the SINR of SU. At the first step, it is assumed that $\mathbf{u}_{p}$ and $\mathbf{u}_{s}$ vectors and also the power of relay node, $P_{r_{k}}$, are known. So $\mathbf{v}_{p}$ and $\mathbf{v}_{s}$ vectors are estimated by maximizing the criterion of (6). From (5), by using the following eigenvalue decomposition and also relationship (8) in the bottom of the page

$$
P_{r_{k}} \mathbf{H}_{s r_{k}}^{H} \mathbf{H}_{r_{k} s}^{H} \mathbf{u}_{s} \mathbf{u}_{s}^{H} \mathbf{H}_{r_{k} s} \mathbf{H}_{s r_{k}}=\mathbf{Q}_{s} \boldsymbol{\Lambda}_{s} \mathbf{Q}_{s}^{H}
$$

we can rewrite the objective function as follows

$$
\begin{aligned}
& \underset{\mathbf{v}_{s}, \mathbf{u}_{s}, \mathbf{v}_{p}, \mathbf{u}_{p}}{\arg \max }\left\{\frac{\mathbf{v}_{s}^{H} \mathbf{Q}_{s} \boldsymbol{\Lambda}_{s} \mathbf{Q}_{s}^{H} \mathbf{v}_{s}}{\mathbf{v}_{p}^{H} \mathbf{Q}_{p} \boldsymbol{\Lambda}_{p} \mathbf{Q}_{p}^{H} \mathbf{v}_{p}}\right\} \\
& \left(P_{r_{k}} \mathbf{H}_{p r_{k}}^{H} \mathbf{H}_{r_{k} s}^{H} \mathbf{u}_{s} \mathbf{u}_{s}^{H} \mathbf{H}_{r_{k} s} \mathbf{H}_{p r_{k}}+\mathbf{H}_{p s}^{H} \mathbf{u}_{s} \mathbf{u}_{s}^{H} \mathbf{H}_{p s}\right)+\left(\frac{N_{s}+P_{r_{k}} N_{r_{k}}}{P_{p}}\right) \mathbf{I}_{N_{p}}=\mathbf{Q}_{p} \boldsymbol{\Lambda}_{p} \mathbf{Q}_{p}^{H} \\
& p_{p}\left|\mathbf{w}_{p}^{H}\left(\mathbf{H}_{p}^{H}+\sqrt{P_{r_{k}}} \mathbf{H}_{p r_{k}}^{H} \mathbf{H}_{r_{k} p}^{H}\right) \mathbf{u}_{p}\right|^{2}-p_{s} \gamma_{p}\left|\mathbf{w}_{s}^{H}\left(\mathbf{H}_{s p}^{H}+\sqrt{P_{r_{k}}} \mathbf{H}_{s r_{k}}^{H} \mathbf{H}_{r_{k} p}^{H}\right) \mathbf{u}_{p}\right|^{2}-p_{r_{k}} \gamma_{p}\left|\mathbf{w}_{r_{k}}^{H} \mathbf{H}_{r_{k}}^{H} \mathbf{u}_{p}\right|^{2}=\left(N_{p}+P_{r_{k}} N_{r_{k}}\right) \gamma_{p} \\
& \operatorname{SINR}_{\mathbf{S U}}=\frac{P_{r_{k}} \mathbf{u}_{s}^{H} \mathbf{H}_{r_{k} s} \mathbf{H}_{s r_{k}} \mathbf{v}_{s} \mathbf{v}_{s}^{H} \mathbf{H}_{s r_{k}}^{H} \mathbf{H}_{r_{k} s}^{H} \mathbf{u}_{s}}{\mathbf{u}_{s}^{H}\left(\mathbf{H}_{p s} \mathbf{v}_{p} \mathbf{v}_{p}^{H} \mathbf{H}_{p s}^{H}+P_{r_{k}} \mathbf{H}_{r_{k} s} \mathbf{H}_{p r_{k}} \mathbf{v}_{p} \mathbf{v}_{p}^{H} \mathbf{H}_{p r_{k}}^{H} \mathbf{H}_{r_{k} s}^{H}+N_{s}+P_{r_{k}} N_{r_{k}}\right) \mathbf{u}_{s}} \\
& \mathbf{H}_{p s} \mathbf{v}_{p} \mathbf{v}_{p}^{H} \mathbf{H}_{p s}^{H}+P_{r_{k}} \mathbf{H}_{r_{k} s} \mathbf{H}_{p r_{k}} \mathbf{v}_{p} \mathbf{v}_{p}^{H} \mathbf{H}_{p r_{k}}^{H} \mathbf{H}_{r_{k} s}^{H}+N_{s}+P_{r_{k}} N_{r_{k}}=\Upsilon_{s}^{H} \Upsilon_{s}
\end{aligned}
$$


By using the decomposition in (16) in the bottom of the previous page and defining $\mathbf{f}_{s}=\Upsilon_{s} \mathbf{u}_{s}$, the objective function becomes

$$
C=\underset{\mathbf{v}_{s}, \mathbf{u}_{s}, \mathbf{v}_{p}, \mathbf{u}_{p}}{\arg \max }\left\{\frac{\mathbf{f}_{s}^{H} \Upsilon_{s}^{-H} P_{r_{k}} \mathbf{H}_{r_{k} s} \mathbf{H}_{s r_{k}} \mathbf{v}_{s} \mathbf{v}_{s}^{H} \mathbf{H}_{s r_{k}}^{H} \mathbf{H}_{r_{k} s}^{H} \Upsilon_{s}^{-1} \mathbf{f}_{s}}{\mathbf{f}_{s}^{H} \mathbf{f}_{s}}\right\}
$$

To maximize the criterion of the proposed algorithm, $\mathbf{u}_{s}$ should be proportional to the eigenvector of $\Upsilon_{s}^{-H} P_{r_{k}} \mathbf{H}_{r_{k} s} \mathbf{H}_{s r_{k}} \mathbf{v}_{s} \mathbf{v}_{s}^{H} \mathbf{H}_{s r_{k}}^{H} \mathbf{H}_{r_{k} s}^{H} \Upsilon_{s}^{-1}$ corresponding to the maximum eigenvalue of $\Upsilon_{s}^{-H} P_{r_{k}} \mathbf{H}_{r_{k} s} \mathbf{H}_{s r_{k}} \mathbf{v}_{s} \mathbf{v}_{s}^{H} \mathbf{H}_{s r_{k}}^{H} \mathbf{H}_{r_{k} s}^{H} \Upsilon_{s}^{-1}$ and that we note it $\mathbf{g}_{s}^{\max }$.

Since the $\Upsilon_{s}^{-H} P_{r_{k}} \mathbf{H}_{r_{k} s} \mathbf{H}_{s r_{k}} \mathbf{v}_{s} \mathbf{v}_{s}^{H} \mathbf{H}_{s r_{k}}^{H} \mathbf{H}_{r_{k} s}^{H} \Upsilon_{s}^{-1}$ matrix have only one non-zero eigenvalue, $\mathbf{u}_{s}$ become

$$
\mathbf{u}_{s}=\frac{1}{\left\|\left(\mathbf{\Upsilon}_{s}^{H} \mathbf{\Upsilon}_{s}\right)^{-1} \mathbf{H}_{r_{k} s} \mathbf{H}_{s r_{k}} \mathbf{v}_{r_{k}}\right\|}\left(\mathbf{\Upsilon}_{s}^{H} \Upsilon_{s}\right)^{-1} \mathbf{H}_{r_{k} s} \mathbf{H}_{s r_{k}} \mathbf{v}_{r_{k}}
$$

To estimate $\mathbf{u}_{p}$, from (4) and the PU's QoS guarantee constraint in (6), we can rewrite the PU's SINR constraint as (19) in the bottom of the page. By using the decomposition in (20) in the bottom of the page, we can obtain $\mathbf{u}_{p}$ as

$$
\mathbf{u}_{p}=\mathbf{g}_{\min }
$$

where $\mathbf{g}_{\min }$ is the eigenvector of $\mathbf{G}_{p} \boldsymbol{\Sigma}_{p} \mathbf{G}_{p}^{H}$ corresponding to the minimum eigenvalue of $\mathbf{G}_{p} \boldsymbol{\Sigma}_{p} \mathbf{G}_{p}^{H}$. Note that $p_{p}, p_{s}$ and $p_{r_{k}}$ (for $k=1, \cdots, K$ ) have been chosen in (12) to (14) such that the left hand side of (19) does not become negative value.

The proposed algorithm is estimated transmitter and receiver beamforming vectors of the PU and SU based on the two-step algorithm in an iterative manner. After power allocation and beamformers estimation phase, the relay is selected based on the maximum SINR of the SU. To do so, the SINR of the SU is computed for each of relay nodes and the relay node with the maximum value of SU's SINR is selected. So the relay selection phase is formulated as follows.

$$
k^{*}=\underset{k}{\arg \max }\left\{\operatorname{SINR}_{\mathrm{SU}}\right\}
$$

\section{COMPUTER Simulation Results}

In this section, simulation results are presented to evaluate the performance of the proposed cooperative beamforming, power allocation and relay selection algorithm. In the simulations, we use flat Raleigh fading MISO and MIMO channels for PU, SU and relay node such that the elements of

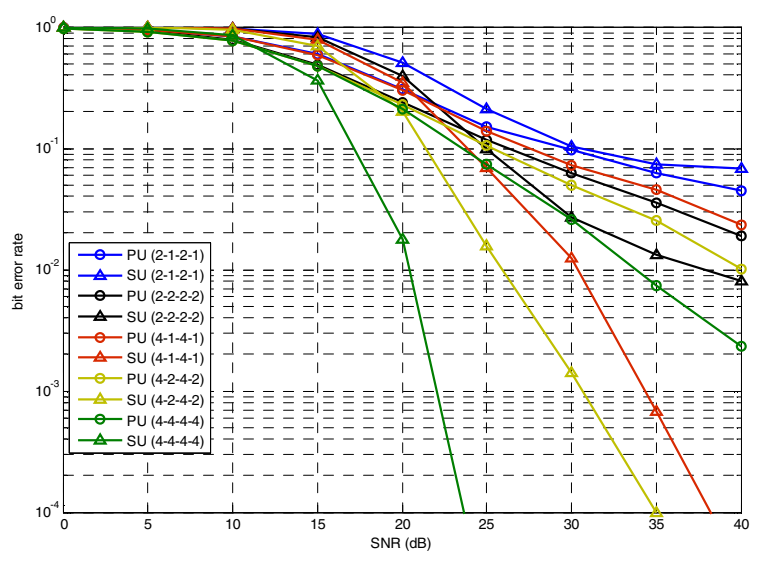

Fig. 2 The BER performances of the PU and SU in MISO and MIMO systems with different number of antennas.

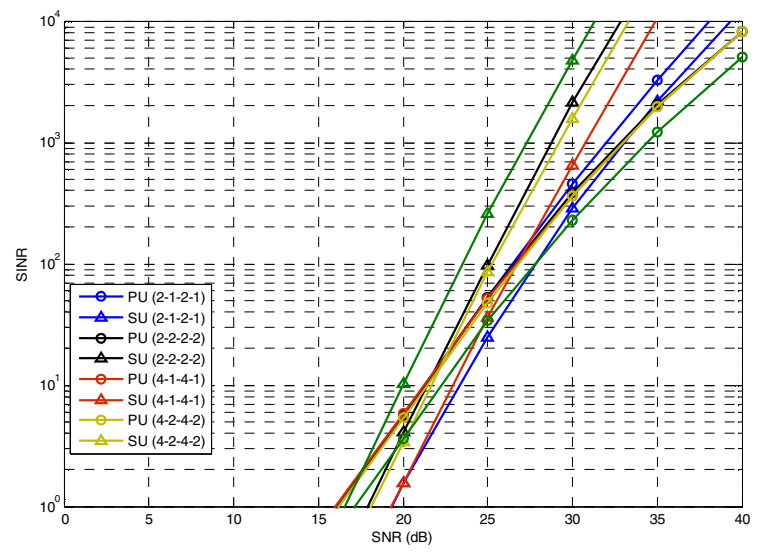

Fig. 3 The SINRs of the PU and SU in MISO and MIMO systems with different number of antennas.

the channel matrices are independent and have normal zero mean Gaussian distributions. The modulation is QPSK and results are obtained for 10000 realizations of channels. In the figures, the number of antennas used in the PU and SU are indicated, respectively, based on PU-Tx, PU-Rx, SU-Tx and SU-Rx array. Meanwhile, the threshold value of $\mathrm{SINR}_{\mathrm{PU}}$ is $\gamma_{p}=5 \mathrm{~dB}$ in simulations.

The PU's and SU's bit error rate (BER) and SINR are shown in Fig. 2 and Fig. 3, respectively for MISO and MIMO channels with different number of antennas. As it's seen, in low SNRs, to guarantee the QoS of PU, only PU is serviced, but in high SNRs the BER performances of SUs are decreased. The BER of the PU is decreased when no power is allocated to the SUs; for example in 4-1-4-1 situation, no power is allocated to SUs before $\mathrm{SNR}=10 \mathrm{~dB}$, thus the BER of the PU is improved by increasing the SNR.

$$
\begin{aligned}
& \mathbf{u}_{p}^{H}\left[\left(\mathbf{H}_{p}+\sqrt{P_{r_{k}}} \mathbf{H}_{r_{k} p} \mathbf{H}_{p r_{k}}\right) \mathbf{v}_{p} \mathbf{v}_{p}^{H}\left(\mathbf{H}_{p}^{H}+\sqrt{P_{r_{k}}} \mathbf{H}_{r_{r_{k}}}^{H} \mathbf{H}_{r_{k} p}^{H}\right)-\gamma_{p}\left(\mathbf{H}_{s p}+\sqrt{P_{r_{k}}} \mathbf{H}_{r_{k} p} \mathbf{H}_{s r_{k}}\right) \mathbf{v}_{s} \mathbf{v}_{s}^{H}\left(\mathbf{H}_{s p}^{H}+\sqrt{P_{r_{k}}} \mathbf{H}_{s r_{k}}^{H} \mathbf{H}_{r_{k} p}^{H}\right)-\gamma_{p}\left(N_{p}+P_{r_{k}} N_{r_{k}}\right) \mathbf{I}_{M_{p}}\right] \mathbf{u}_{p} \geq 0 \\
& \left(\mathbf{H}_{p}+\sqrt{P_{r_{k}}} \mathbf{H}_{r_{k} p} \mathbf{H}_{p r_{k}}\right) \mathbf{v}_{p} \mathbf{v}_{p}^{H}\left(\mathbf{H}_{p}^{H}+\sqrt{P_{r_{k}}} \mathbf{H}_{p r_{k}}^{H} \mathbf{H}_{r_{k} p}^{H}\right)-\gamma_{p}\left(\mathbf{H}_{s p}+\sqrt{P_{r_{k}}} \mathbf{H}_{r_{k} p} \mathbf{H}_{s r_{k}}\right) \mathbf{v}_{s} \mathbf{v}_{s}^{H}\left(\mathbf{H}_{s p}^{H}+\sqrt{P_{r_{k}}} \mathbf{H}_{s r_{k}}^{H} \mathbf{H}_{r_{k} p}^{H}\right)-\gamma_{p}\left(N_{p}+P_{r_{k}} N_{r_{k}}\right) \mathbf{I}_{M_{p}}=\mathbf{G}_{p} \boldsymbol{\Sigma}_{p} \mathbf{G}_{p}^{H}
\end{aligned}
$$


The BER of the PU is decreased when no power is allocated to the SUs; for example in 4-1-4-1 situation, no power is allocated to SUs before $\mathrm{SNR}=10 \mathrm{~dB}$, thus the BER of the PU is improved by increasing the SNR. For SNR $\geq 20 \mathrm{~dB}$, the BER performance of the SU outperforms the BER of the PU. Note that in all situations, the performance of the PU is equal or larger than the threshold such that at high SNRs, the BER performances of SUs are decreased rapidly.

\section{CONCLUSION}

A cooperative beamforming, power allocation and relay selection algorithm has been proposed in this paper for MIMOrelay cognitive radio systems. The proposed algorithm estimates the transmitter and receiver beamforming vectors of primary user (PU) and secondary user (SU) along with allocated powers to them. In this algorithm, SINR of SU is maximized under a maximum allowable transmit power of SU and relay node and also a threshold SINR of the PU constraints. The performance of the algorithm has been evaluated by computer simulations. The results indicated that the proposed algorithm, in addition to guarantee a required performance of the PU, increases spectrum usage efficiency by servicing the SU.

\section{ACKNOWLEDGMENT}

The authors would like to thank Iran Research Institute for ICT for partially supporting this work.

\section{REFERENCES}

[1] S. Haykin, "Cognitive radio: Brain-empowered wireless communications" IEEE Trans. Selected Areas in Communications, Vol. 23, No. 2, pp. 201-220, 2005.

[2] S. Srinivasa and S. Jafar, "The throughput potential of cognitive radio: A theoretical perspective," IEEE Asilomar Conf. Signals, Systems and Computers, Pacific Grove, CA, Nov. 2006, pp. 221-225.

[3] M. Shaat and F. Bader, "Asymptotically Optimal Resource Allocation in OFDM-Based Cognitive Networks with Multiple Relays" IEEE Trans. Wireless Communications, Vol. 11, No. 3, pp. 892-897, 2012.

[4] G. Zhao and Ch. Yang and G. Y. Li and D. Li and A. C. K. Soong, "Power and Channel Allocation for Cooperative Relay in Cognitive Radio Networks" IEEE J. Selected Topics in Signal Processing, Vol. 5, No. 1, pp. 151-159, 2011.

[5] D. Bharadia and G. Bansal and P. Kaligineedi and V. K. Bhargava, "Relay and Power Allocation Schemes for OFDM-Based Cognitive Radio Systems" IEEE Trans. Wireless Communications, Vol. 10, No. 9, pp. 2812-2817, 2011.

[6] Y. Pan and A. Nix and M. Beach, "Distributed Resource Allocation for OFDMA-Based Relay Networks" IEEE Trans. Vehicular Technology, Vol. 60, No. 3, pp. 919-930, 2011.

[7] L. Li and X. Zhou and H. Xu and G. Y. Li and D. Wang and A. Soong, "Simplified Relay Selection and Power Allocation in Cooperative Cognitive Radio Systems" IEEE Trans. Wireless Communications, Vol. 10, No. 1, pp. 33-36, 2011.

[8] P. Ubaidulla and Sonia Aissa, "Optimal Relay Selection and Power Allocation for Cognitive Two-Way Relaying Networks" IEEE Wireless Communications Letters, Vol. 1, No. 3, pp. 225-228, 2012.
[9] M. Choi and J. Park and S. Choi, "Simplified Power Allocation Scheme for Cognitive Multi-Node Relay Networks" IEEE Trans. Wireless Communications, Vol. 11, No. 6, pp. 2008-2012, 2012.

[10] J. Tang and S. Lambotharan, "Beamforming and temporal power optimisation for an overlay cognitive radio relay network" IET Journal on Signal Processing, Vol. 5, No. 6, pp. 582-588, 2011.

[11] H. Zamiri-Jafarian and M. Abbasi-Jannatabad, "Cooperative Beamforming and Power Allocation in the Downlink of MIMO Cognitive Radio Systems" IEEE Conf. on Vehicular Technology (VTC 2010-Fall), pp. 1-5, 2010.

[12] M. Abbasi-Jannatabad and H. Zamiri-Jafarian, "Cooperative Beamforming in MIMO-OFDMA Cognitive Radio Systems" IEEE Int. Symp. on Telecommunications (IST 2010), pp. 38-42, 2010. 